Editorial

\section{Con gran emoción}

\section{With Great Excitement}

\author{
D. Mysler ${ }^{1}$ \\ ${ }^{1}$ Instituto Alexander Fleming, Buenos Aires, Argentina
}

Rev Argent Radiol 2018;82:1.

La Sociedad Argentina de Radiología acaba de cumplir 100 años de existencia, siendo la segunda sociedad americana más antigua. Dicen que con los años llega la madurez y la sabiduría al ser humano y por qué no también a las sociedades. Vemos por ello y con sumo agrado, el restablecimiento de las relaciones entre SAR y FAARDIT, con la idea directriz de sentar las bases para que ambas trabajen y se potencien juntas por otros 100 años.

Luego de muchos años de gran desarrollo y confraternidad de toda la radiología en general y de las sociedades en particular, sobrevinieron algunos años de distanciamiento, seguramente basados en intereses que en dicho momento se consideraron genuinos y de buena fe para las autoridades conductoras, pero que a la luz de los hechos, impactaron negativamente en la radiología argentina. La separación de SAR y FAARDIT hoy es cosa del pasado.

Las fortalezas y oportunidades de progreso de cada una de las entidades potenciarán la misión y visión de toda la radiología argentina, sin por ello dejar de lado el valor de las identidades. Por esa razón, hemos constituido acuerdos de cooperación mutua que beneficiarán en forma ecuánime a ambas sociedades.

Estamos orgullosos y satisfechos de emprender el desafío de volver a contar con una Revista Argentina de Radiología unida, trabajando en equipo para lograr una publicación de excelencia, sumando los valores de todos los radiólogos.

Algunas actividades serán nuevamente compartidas de aquí en adelante por SAR y FAARDIT, enriqueciendo y jerarquizando la calidad, el diseño y el currículum. Podemos citar como ejemplos: la RAR, el CONAEDI, el CADI, los congresos internacionales y algunos cursos virtuales nacionales y latinoamericanos.

La SAR perdurará con su autonomía, sus asociados y sus lineamientos particulares de una gran familia radiológica ya centenaria, así como también lo hará FAARDIT. Hemos hecho hincapié en el pilar básico de la sociedad, la educación médica continua, trabajando en todas las áreas programáticas del postgrado.

Dentro de los valores que hemos querido estimular, nos volcamos a los jóvenes. A ellos destinamos gran parte del presupuesto societario, renovando completamente el Curso Superior que otorga los certificados habilitantes para la práctica de la radiología, el rediseño de los cursos virtuales y presenciales, otorgando becas de formación, asistencia y participación en los congresos nacionales y en las actividades internacionales. Nos hemos avocado especialmente a definir los criterios de selección a la hora de otorgar esos beneficios a nuestros jóvenes radiólogos, privilegiando la idoneidad, el talento y la igualdad de oportunidades.

Haciendo foco en el futuro y en la búsqueda de nuevos líderes para nuestra sociedad decidimos crear una comisión de profesionales jóvenes, surgida de aquellos radiólogos que egresan de nuestro Curso Superior. Esta comisión se reúne desde hace más de un año para debatir y emitir opiniones y sugerencias para la comisión directiva, otorgando una mirada lozana con puntos de vista originales que intentan conducirnos hacia la radiología que los jóvenes esperan en el mañana que ellos transitarán.

Desde SAR y FAARDIT trabajaremos incansablemente en conjunto en todas las áreas donde la radiología argentina se fortalezca con la unidad, hacia adentro y fundamentalmente hacia afuera, priorizando la transparencia, la ética en la gestión, la rectitud y la buena fe de sus integrantes. Naturalmente no siempre estaremos de acuerdo, pero el debate y la búsqueda de consensos arribarán siempre a soluciones equilibradas y acertadas para la radiología argentina en general.

Estamos culminando el primer mandato de este grupo de profesionales decididos a emprender un cambio de timón en el cual impere el diálogo, la camaradería, el pluralismo de ideas, el trabajo en equipo, el intercambio intersocietario para generar valor, y el compromiso con la transparencia y la ética. La continuidad de este proyecto permitirá afianzar lazos y construir nuevos puentes entre las sociedades que harán aún más fuerte los vínculos actuales y permitirán proyectos más ambiciosos que beneficiarán seguramente a todos los radiólogos de nuestro país.

Es por todo ello que, con este espíritu, inauguramos este nuevo año y damos la bienvenida a la unificada RAR de SAR y FAARDIT.

Dr. Daniel Mysler

Vicepresidente

Sociedad Argentina de Radiología

\author{
Address for correspondence \\ Daniel Mysler, MD, Instituto \\ DOI https://doi.org/ \\ $10.1055 / \mathrm{s}-0038-1637723$ \\ Alexander Fleming, Buenos Aires, ISSN 1852-9992. \\ Argentina \\ (e-mail: dmysler@gmail.com).
}

Copyright @ 2019, Sociedad Argentina
de Radiología. Publicado por Thieme terms
Revinter Publicações Ltda., Rio de Janeiro, Brazil. Todos los derechos reservados. 